\title{
Calibration-less quantitative 4D-STEM imaging of amorphous samples
}

\author{
Radim Skoupy and Vladislav Krzyzanek
}

Institute of Scientific Instruments of the Czech Academy of Sciences, Czech Republic, Brno, Jihomoravsky kraj, Czech Republic

The regular quantitative scanning transmission electron microscopy (STEM) technique uses a detector calibration process as a bridge between captured image of a sample and computer simulation of electron scattering in a model sample with known geometry and composition [1,2]. Unfortunately, this approach needs capturing of two calibration images before each imaging session and it is probe current, acceleration voltage and contrast/brightness dependent.

We present a simple method for quantitative imaging using 2D STEM pixelated detector. In this case, no special calibration is needed. 4D datasets captured by the 2D STEM detectors are often used for many imaging and analytical techniques including thickness measurement in the field of material science and diffracting samples [3] or less frequently used for analysis of amorphous samples [4].

The presented 4D-qSTEM method is based on shifting of the most common scattering angle to the higher angles with increasing sample thickness. As the primary electron beam is scanned over a sample (Fig. 1a), individual scattering maps are captured for each beam position. Individual pixels are summarised by their distance from the centre (Fig. 1b) and plotted accordingly to its angle based on the detector/sample geometry. The computational inputs of the method are similar dependencies based on Monte Carlo simulation in a sample with known thickness, density and composition. At both parts, the most probable scattering angle is found and local thickness is estimated by finding of experiment-based angle in simulation-based series.

In the first experiments, latex nanospheres with a diameter of $575 \mathrm{~nm}$ (Fig. 2a) were chosen as a model sample for their well-known dimensions at every point. From the comparison of the experimental scattering angle dependency and the simulation, the best match was found for the thickness of $555 \mathrm{~nm}$ (Fig. 2b). The error of estimation is around $3.5 \%$ according to the measured nanosphere diameter, but it could be caused by the imperfectly round shape of the nanosphere.

The main advantage of the method is that no previous detector calibration is needed. The usable range of the thicknesses which can be determined is given by the creation of detectable peak at low scattering angles for low thicknesses. In the case of latex, the measurable range is from $200 \mathrm{~nm}$ to $1000 \mathrm{~nm}$. We suggest that this method can enrich a number of methods based on pixelated STEM detectors and extend their use on amorphous types of samples.

\section{Acknowledgement}

The research was supported by the Czech Science Foundation (project 21-13541S). The authors thank Thermo Fisher Scientific Brno for providing the FIB-SEM Helios equipped with the T-pix detector (based on TimePix technology) as a part of the Technology Agency of the Czech Republic project (TN01000008). 

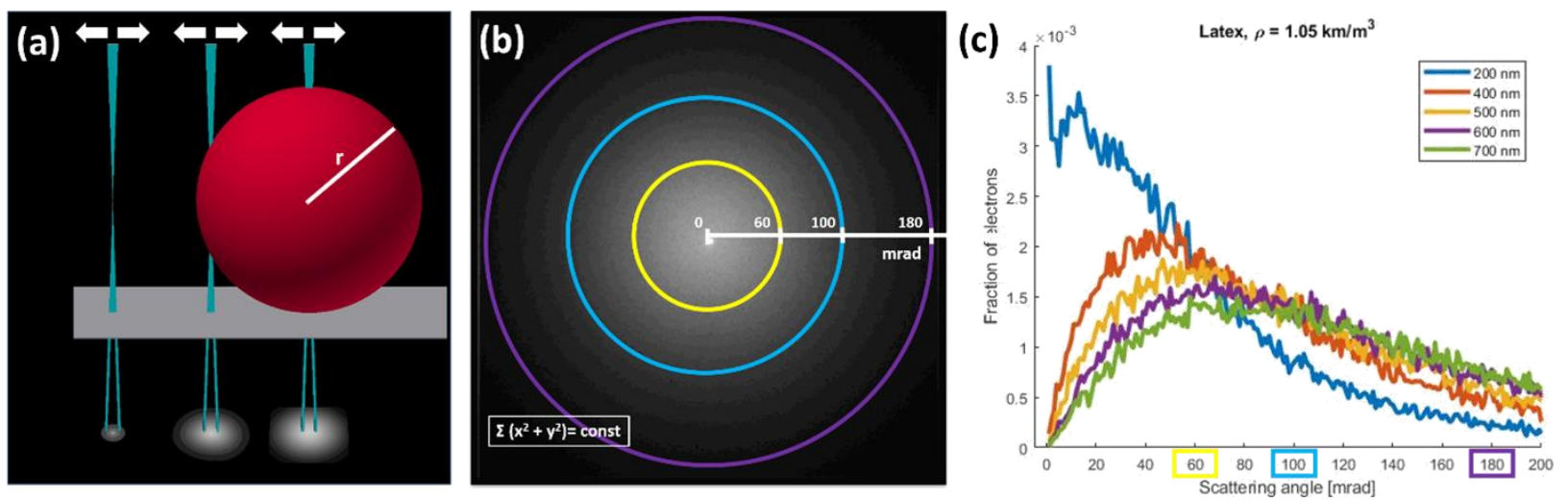

Figure 1. Principe of 4D-qSTEM. (a) Test sample of Latex nanosphere with radius $r$ is irradiated by scanning electron beam. Scattering patterns are captured independently for all beam positions. (b) The rotation sum of captured scattering pattern is computed and the local dependency of primary electron fraction is plotted accordingly to the scattering angle. (c) Simulated scattering angle dependency for several sample thicknesses. Note that the position of the peak is moving to higher angles with increasing thickness.
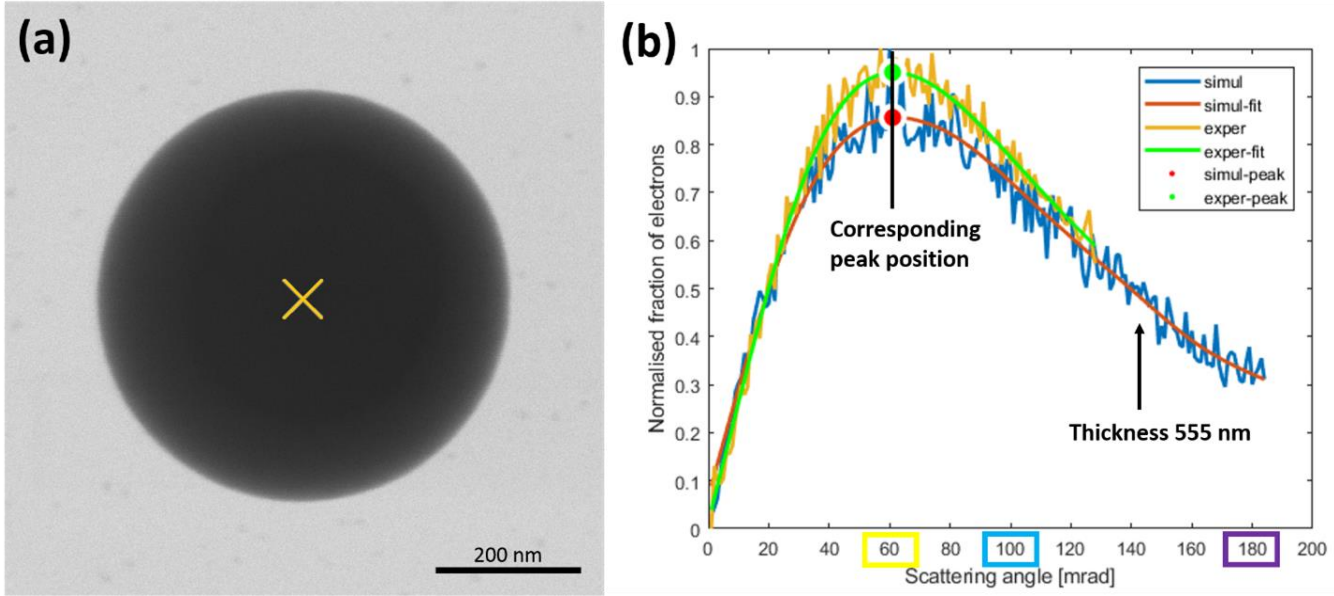

Figure 2. (a) Test sample of Latex nanosphere with a diameter of $575 \mathrm{~nm}$ with the measured point in the middle of the sphere. (b) Comparison of scattering angle dependencies from the measured point and simulation for $555 \mathrm{~nm}$ thick layer of latex. The position of both peaks is at $61 \mathrm{mrad}$.

\section{References}

[1] R. Skoupy, J. Nebesarova, M. Slouf, V. Krzyzanek, Quantitative STEM imaging of electron beam induced mass loss of epoxy resin sections, Ultramicroscopy. 202 (2019) 44-50. doi:10.1016/j.ultramic.2019.03.018.

[2] M. Pfaff, E. Müller, M.F.G. Klein, A. Colsmann, U. Lemmer, V. Krzyzanek, R. Reichelt, D. Gerthsen, Low-energy electron scattering in carbon-based materials analyzed by scanning transmission electron microscopy and its application to sample thickness determination, J. Microsc. 243 (2011) 31-39. doi:10.1111/j.1365-2818.2010.03475.x.

[3] C. Ophus, Four-Dimensional Scanning Transmission Electron Microscopy (4D-STEM): From Scanning Nanodiffraction to Ptychography and Beyond, Microsc. Microanal. (2019) 563-582. doi:10.1017/S1431927619000497.

[4] X. Mu, A. Mazilkin, C. Sprau, A. Colsmann, C. Kübel, Mapping structure and morphology of amorphous organic thin films by 4D-STEM pair distribution function analysis, Microscopy. 68 (2019) 301-309. doi:10.1093/jmicro/dfz015. 